\title{
ART, MUSIC, AND LITERATURE: DO THE HUMANITIES MAKE OUR LIVES RICHER, HAPPIER, AND MORE MEANINGFUL?
}

\author{
Erin C. Westgate, Shigehiro Oishi
}

\begin{abstract}
For many, there is little more rewarding than the feeling of curling up with a good book, wandering a famous art gallery, or listening to a favorite musician perform live in front of an audience. But do the arts, music, and literature actually make our lives happier, richer, and more meaningful? We suggest they do. In this chapter, we review empirical evidence for the psychological benefits of the humanities, including art, music, and literature, and find that across a wide variety of samples, exposure and engagement is consistently linked to greater well-being. In particular, we suggest that the humanities may increase well-being directly by providing people with enjoyable, rich, and meaningful experiences, as well as indirectly by fostering skills and abilities that contribute to psychological well-being in the long-term. These approaches map onto two mechanisms: 1) direct affective benefits that create enjoyable, rich, and interesting experiences, and 2) indirect cognitive benefits, including social abilities and motivations that promote subjective well-being via interpersonal connection and self- and emotion-regulation. Art, music, and literature may not only provide temporary nourishment for a good life, but teach people lasting skills they can capitalize on to increase longterm well-being.
\end{abstract}

Keywords: Happiness, Psychological Richness, Subjective Well-being, Arts, Music, Humanities, Literature, Theater, Dance, Novels, Interest

In an iconic moment in the second half of Handel's Messiah, the audience - until this moment politely seated and attentive - traditionally rises to its feet in a surge of rustling suits and fabric, as the first four notes sound the start of the "Hallelujah Chorus". As the triumphant notes ring out from the chorus, the audience joins them silently, standing together in solidarity in a moment that for many audiences is both exciting and profoundly moving. Certainly it seems plausible that such transcendent moments enrich our lives, and make them happier, richer, and more meaningful. In this chapter, we explore empirical evidence for the psychological benefits of the humanities, including art, music, and literature.

\section{Why the humanities?}

Why might literature and the performing and visual arts increase subjective well-being? We suggest that the humanities can increase well-being directly, as well as indirectly foster skills and abilities that contribute to psychological well-being in the long-term. Art, music, and literature may not only provide temporary nourishment for a good life, but teach people lasting skills they can capitalize on to increase long-term well-being.

These approaches map onto two mechanisms: 1) direct affective benefits that create enjoyable, rich, and interesting experiences, and 2) indirect cognitive benefits, including social abilities and motivations that promote subjective well-being via interpersonal connection and self- and emotion-regulation (e.g., Shim et al., 2019).

\section{Do art, music, and literature make our lives better?}

Before turning to the question of why the humanities might improve our lives, what is the empirical evidence that art, music, and literature improve our lives in the first place?

Happiness and life satisfaction. The arts and humanities are associated with greater life happiness in a 
wide variety of cross-sectional samples. In data from over 48,000 people in 33 countries, time spent reading, listening to music, and attending cultural events are all positively associated with greater happiness (Wang \& Wong, 2014). Similar results are found in individual countries. Cultural activities (e.g., reading, theater, concerts, etc.) are positively associated with subjective well-being among 20,000+ adults in the German SocioEconomic panel (Frey, 2019) as well as in a nationally representative sample of Croatians (at least for those over 30; Brajša-Žganec et al., 2011). Likewise, literature, music, and the arts, are all modestly associated with greater well-being among Italians (Blessi et al., 2014).

Even small doses of the humanities may be beneficial. Whereas only regular engagement in the arts (i.e., at least weekly) predicted life satisfaction in 30,000 British residents, attending arts events and cultural sites (e.g., museums, monuments) was positively related to happiness and life satisfaction, even among people who rarely went (Wheatley \& Bickerton, 2017). And while concerts and the cinema predicted greater life satisfaction among 10,000 British individuals, effects were stronger for people who attended less often (Marsh \& Bertranou, 2012).

Different activities may be beneficial in different doses. For instance, more frequent attendance of musical cultural activities (e.g., jazz, opera/ballet, classical concerts) was associated with greater well-being among Italians (up to 100 times a year); but attending too often blunted the positive effects of poetry reading and art cinema (Gross et al., 2011). Likewise, in longitudinal studies, people who upped their attendance at arts events and historical sites over a three year period reported corresponding increases in life satisfaction; yet the same increases in arts activities had no effect (and increased library visits a negative effect) on life satisfaction (Wheatley \& Bickerton, 2019). One alternative is that quantity may matter less than type and variety of experience; for instance, more frequent and more diverse cultural experiences (including literature, painting, art, theater, and dance) predicted happiness and life satisfaction in Korea (Kim \& Kim, 2008).

Although the above studies are correlational, experimental evidence suggests such effects may be causal. For instance, expressive writing increases people's subjective well-being (Pennebaker, 1997), and older adults randomly assigned to a theater arts intervention reported greater psychological well-being, relative to a visual arts appreciation intervention or wait-list control (Noice, Noice, \& Staines, 2004). Likewise, students randomly assigned to take personally meaningful photographs reported increases in global life satisfaction, as well as meaning (Steger et al., 2013), and nursing staff assigned to a 10 -week silk painting class reported greater emotional well-being (Karpavičiūtė \& Macijauskienè, 2016). Music appears likewise beneficial older adults randomly assigned to sing in a choir reported higher quality of life, and reduced depression and anxiety, three months later (Clift et al., 2012), and those randomly assigned to a six-month dance intervention experienced higher psychological well-being (Kattenstroth et al., 2013). Although fewer studies have examined the visual arts, art therapy appears effective. For instance, older adults randomly assigned to an art therapy class with painting and clay art reported less depression and anxiety (Kim, 2013), and, in a metaanalysis, creative art therapy was found to effectively reduce anxiety and depression in cancer patients, and improve their quality of life (Puetz et al., 2013).

Psychological richness. Even when the humanities don't make lives happier, they may transform them in other ways - for instance, by making lives psychologically richer. Psychological richness stems from novel complex experiences that change people's view of the world and their place within it; while not always positive, such experiences can form an integral part of what it means to lead a good life (Oishi et al., 2019).

The arts, music, and literature, may offer one such path. For instance, Germans assigned to read Der Vorleser [The Reader] about a man struggling to make sense of his adolescent love affair in post-WW2 Germany with a much older woman (who he, and the reader, later discover to have been a Nazi concentration camp guard) reported declining happiness, but greater "delight" in reading over the course of the novel (van Peer et al., 2007). Such emotional shifts may be typical of complex perspective-changing experiences that, while not always comfortable, are psychologically rich for those immersed in them. For example, consider the visual arts. Some pieces of art are more complex and evoke more change in perspective than others. Figureground drawings offer one such literal example. The same illustration can portray an image of the crescent moon or of an upward-tilted face (see Figure 1, Panel B), depending on whether you focus on the figure versus ground element of the drawing. In a series of experiments, such figure-ground drawings (Panel B of Figure 1) evoked greater psychological richness than the same drawings modified to remove the element that made such dual perspectives possible (Panel A of Figure 1; Oishi \& Choi, 2019). Some art makes the familiar unfamiliar, and the unfamiliar familiar; such shifts 
A

B
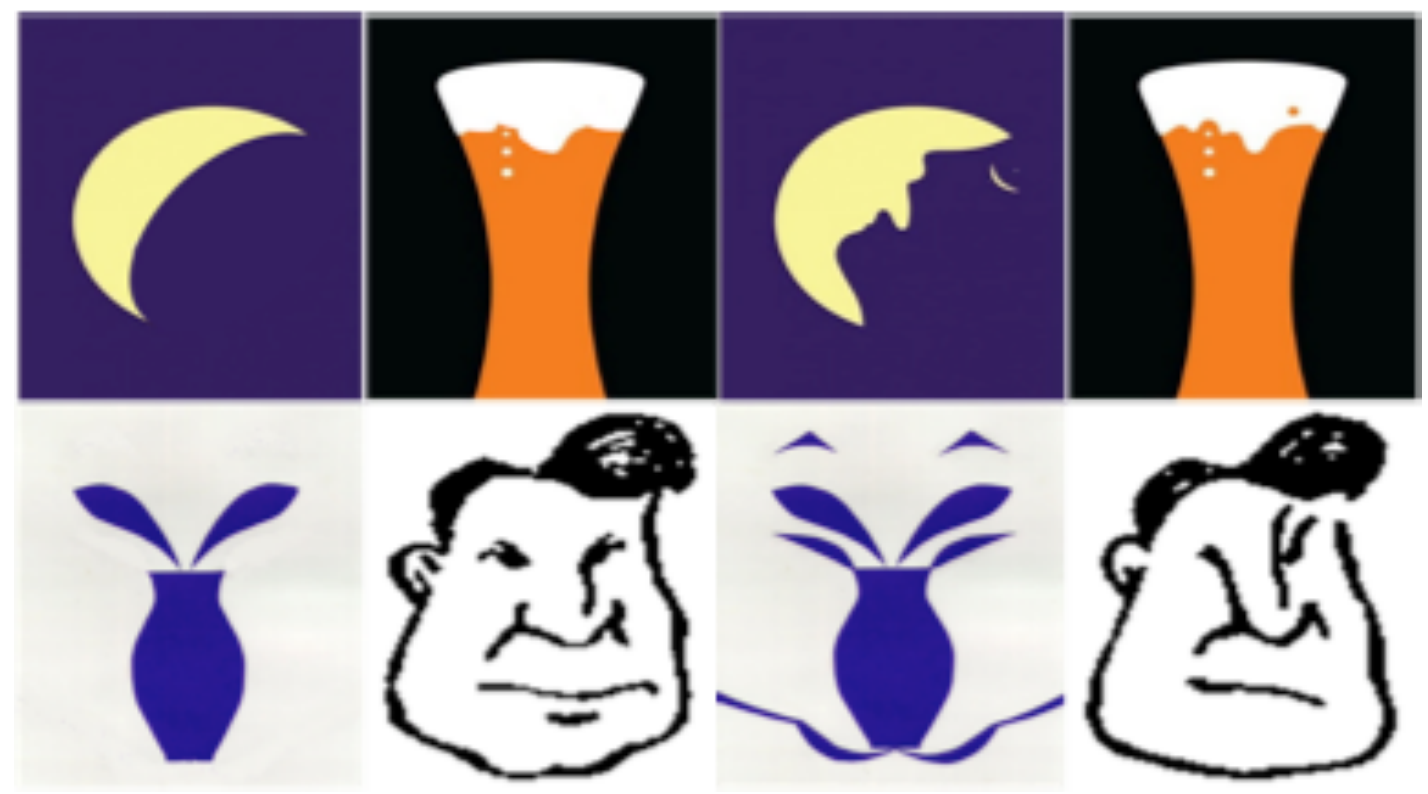

Figure 1. Panel A shows 4 drawings that evoke only a single image (e.g., a moon), whereas Panel B shows 4 figure-ground drawings that evoke two different images (e.g., either a moon or a face); used in Oishi \& Choi (2019, Experiments $1 \& 2$ ). Participants were randomly assigned to one of the two image sets.

in perspective may be key to enriching our daily experiences.

\section{Why do art, music, and literature make our lives better?}

We suggest that the humanities lead to greater wellbeing both directly and indirectly, by providing both affectively rich positive experiences, as well as cognitive skills and social strengths that contribute indirectly to psychological richness and happiness. In their theoretical framework, Tay et al. (2018) likewise divide the humanities' positive outcomes into two distinct sets: 1) positive neurological, physiological, and affective changes, and 2) positive effects on psychological competencies, respectively. What is the evidence for such benefits?

\section{Affective (direct) benefits of the humanities}

Perhaps the simplest question is whether the arts, music, and literature make us happier in the moment, setting aside the question temporarily of whether such affective gains translate into richer or happier lives. To be sure, they likely do - rich and happy lives are built on the building blocks of rich and happy experiences. But what is the evidence that the humanities contribute to those experiences?

In a UK experience sampling study, attending "theatre, dance, concert" was the second happiest activity people reported, followed by visits to an "exhibition, museum, library"; only love-making made people happier (Bryson \& MacKerron, 2017). Likewise, highly educated Germans reported that they were most likely to experience flow - an enjoyable immersive state (Czikszentmihalyi, 2000) - while reading, compared to all other activities (including sex; van Peer, Mentjes, \& Auracher, 2007; see however the null effect among American teenagers, Csikszentmihalyi \& Hunter, 2003).

Are these effects causal or are people simply more likely to turn to the humanities when they feel good? Experimental mood inductions suggest such activities do change how people feel. Music, film, visual images, and written imagery are widely used in the experimental study of emotion, to deliberately induce positive and negative mood states. And they do so quite effectively (Siedlecka \& Denson, 2018; Zhang et al., 2014).

The humanities may also offer a pathway to psychologically rich experiences. For instance, while listening 
to sad music or filming a depressing documentary may not be pleasant, per se, they offer exactly the psychologically rich worldview-shifting moments that change the way people understand themselves and the world. In a weekly diary study of college students studying abroad, study abroad students engaged in artistic activities (e.g., concerts, museum) more often during a 13week period than those who stayed on campus $(d=$ $1.105)$; the same study abroad students also reported their lives were psychologically richer at the end of the semester $(d=.518$ vs students on campus; cf. $d=.039$ beginning of semester; Oishi, Choi, Liu, \& Kurtz, 2019). The increase in psychological richness was explained in part by study abroad students' increased engagement in artistic activities during that time. Studying abroad in an unfamiliar environment may be stressful and challenge one's values and meaning system; thus, it may not make lives happier or meaningful. Notably, the two groups' life satisfaction did not differ at the beginning or end of the semester Yet, such a worldchanging experience seems to increase richness, in part because it entails substantial cultural artistic engagement and shifts in perspective.

\section{Cognitive (indirect) benefits of the humanities}

In addition to directly increasing positive mood or psychological richness, the humanities might indirectly foster increases in subjective well-being by bolstering abilities and skillsets conducive to well-being more broadly.

Work in older adults suggests the humanities may increase overall cognitive functioning; for instance, retirement home residents randomly assigned to a theater arts intervention showed increases in short-and longterm (but not working) memory capacity and creative problem-solving, relative to a singing intervention or wait-list control group (Noice \& Noice, 2009). Likewise, dance training improves cognitive performance, attentional switching, and reaction times in experimental and quasi-experimental interventions (Coubard et al., 2011; Kattenstroth et al., 2013; Kimura \& Hozumi, 2012). In a small randomized control trial, women experiencing burnout participated in a combined creative arts program for three months; the program reduced alexithymia and increased the women's ability to describe and identify their own emotional states (Viding et al., 2015).

A considerable body of work has focused on the role that reading and fiction play in increasing our ability to understand and relate to others' mental states.
Fiction, specifically, has been argued to improve people's ability to infer others' emotional and cognitive states, by simulating social-cognitive processes and learning via concrete fictional examples (Mar \& Oatley, 2008; Oatley, 2016). To this end, a recent metaanalysis found that, relative to not reading or reading non-fiction, randomly assigning people to read works of fiction led to small but significant improvements in social cognition $(\mathrm{g}=.15)$, including mentalizing, perspective-taking, and emotion identification (DodellDeder \& Tamir, 2018). Oatley (1999) argues that fiction acts as a mental simulator more broadly, allowing us to imagine and simulate counter-factual experiences. And indeed, people who read more fiction show greater functional connectivity in brain areas related to language and mentalizing when listening to literary narratives (Willems \& Hartung, 2019). Likewise, music appears to activate portions of the default mode network associated with conscious streams of thought, mindwandering, and mental simulation (Wilkins et al., 2014).

Finally, the humanities may lead people to experience greater reflectiveness, or the intentional motivation to shift one's own identity, values, and beliefs (Tay et al., 2018). Such motivation may partially account for many of the cognitive benefits outlined above, as well as the social benefits outlined below, that together create long-term sustainable changes in psychological well-being. Reflectiveness, in particular, may foster sustained changes in psychological richness, if people take that impetus for change seriously, and come to see themselves and the world from new perspectives.

\section{Social (indirect) benefits of the humanities}

Engagement in the arts, music, and literature may also spill over into social capital, either via status gains by participating in high prestige activities (e.g., art galas, operas) or through artistic activities that act as markers of shared identity (e.g., street art, folk music). Tay et al. (2018) refer to these as socialisation mechanisms that foster flourishing. Writers bond in writing groups, painters and visual artists form collectives, and people come together to consume the books, artwork, and music they produce, leading to vibrant book clubs, lively artshows, and tight-knit music scenes, that blend enjoyment of the arts with social bonding. Such sharing can intensify positive feelings (Boothby, Clark, \& Bargh, 2014), and may itself serve as a powerful source of well-being. 


\section{Unanswered questions}

Initial evidence suggests that art, music, and literature may confer both immediate affective benefits, as well as indirect cognitive and social benefits. However, it is not yet clear how and whether such processes directly account for global lasting changes in well-being, who benefits most from such engagement, and whether some activities are more beneficial than others.

\section{Is it causal?}

Much of the evidence reviewed above is correlational; while good experimental evidence exists for art, literature, and music's ability to temporarily boost positive moods, there is less evidence for whether they lead to lasting changes in well-being. The same is true, to a lesser extent, of the mechanisms at play; while there is strong consensus that the humanities can directly foster positive emotions, claims regarding their effect on cognition have been more controversial (see Dodell-Deder \& Tamir, 2018). And to date it is unclear whether and how such effects might trickle up and account for increases in well-being more globally. Providing rigorous longitudinal experimental evidence for the direct and indirect causal effects of the humanities on overall subjective well-being would greatly contribute to our understanding.

\section{Who benefits and why?}

Does everyone benefit from the humanities, and if so, do they all benefit equally? For instance, in a study of several thousand British residents, engagement in the arts did predict subjective well-being - but only for those who were relatively unhappy to begin with (Hand, 2018). We suggest that there are likely strong person by environment interactions in the humanities, with certain people benefiting more (or less) from particular activities. Why might this be? For people to cognitively engage in and benefit from any activity, they must have both the motivation and ability to do so successfully (Westgate \& Wilson, 2018). For instance, while time spent per week in creative activities did not predict happiness in two Canadian samples, the quality of that engagement did - satisfaction within many creative domains was modestly correlated with happiness (Michalos \& Kahlke, 2008; Michalos \& Kahlke, 2010). When people are both willing and able to appreciate the humanities, the resulting cognitive engagement is generally experienced as feelings of interest, enjoyment, and flow, or what Tay et al. (2018) term immersion.
Such motivation and ability varies widely across people, and interacts with the nature of the task at hand, whether it's reading a novel or solving algebraic equations.

Motivation. Motivation is important both for exposure to the humanities, and to engage successfully and benefit when exposure occurs. People who are motivated and interested in art, music, and literature are more likely to seek them out. Tay et al. (2018) refer to this as acquisition, or a sense of embeddedness in people's lives; in short, the "socio-cognitive processes that underlie practice, learning, and cultivation." People who feel more embedded in the arts - either through encouragement, a sense of mastery, or past positive experiences - are more likely to benefit from them (p. 4). Does it matter whether motivation is intrinsic or extrinsic? In adolescents, hours spent reading for pleasure (but not required reading) predicts GPA (Ferguson, 2014), and choosing to listen to music for personal reasons is a stronger predictor of global happiness (Morinville et al., 2014). Likewise, default mode network activity is higher when participants view works of art (e.g., paintings, sculptures) that they personally like (Vessell, Starr, \& Rubin, 2012); connectivity is likewise higher when listening to favorite pieces of music (Wilkins et al., 2014).

While extrinsic motivation may suffice to increase exposure to the humanities, we suggest intrinsic motivation is necessary for high-quality engagement, the sort of rich rewarding encounters that increase subjective well-being. People who are motivated to seek out such experiences, and are rewarded affectively with rich and positive experiences in doing so, may be particularly likely to seek out such opportunities again in the future. Indeed, evidence from a longitudinal study of talented teens suggests that only those teens who learned to enjoy practicing stuck with their talent (e.g., art, music, science) in later years (Csikszentmihalyi et al., 1997).

Ability. Motivation matters; yet work in aesthetics suggests that engagement requires that a person cognitively grasp their experience, whether it be poetry, artwork, or a piece of music (Silvia, 2006). For instance, people assigned to read "The Whitest Parts of the Body", a modern abstract poem from The Life of Haifisch, enjoyed it only if they were first clued in that the poem was about sharks (Silvia, 2005). Likewise, in literature, background knowledge and genre expectations shape readers' experiences (Hartung et al., 2017; Hoven et al., 2016). Indeed, one of the best determinants of reading comprehension is not merely the text's difficulty, but how much the reader knows about the 
topic. Pilots do better at reading aviation texts than nonpilots, and baseball fans do better at reading stories about a ball game (Morrow, Leirer, \& Altieri, 1992; Recht \& Leslie, 1988). Similarly, appreciating modern jazz requires understanding the conventions that govern it.

Background knowledge may also enhance psychological richness. In one experiment, participants listened to the pianist Nobuyuki Tsujii perform an etude by Chopin (Oishi \& Axt, 2019). Beforehand, half the participants were informed that Mr. Tsujii was born blind. Despite watching the same performance and learning the same background information (with the exception of Mr. Tsujii's blindness), only participants who knew he was blind reported greater psychological richness; there were no differences in mood. Music often moves us, but with additional information, it may move us even more. For instance, even knowing the background of Chopin's "revolutionary" Etude Op. 10 No. 12 - namely, that it was written as he fled his beloved homeland of Poland for France amidst a failed attempt at revolution, never again to set foot in his home country - might lead to a psychologically richer experience.

In sum, people need to be both able and motivated to benefit from the humanities, if the arts, music, and literature are to enhance their subjective well-being. But whether they are able to do so may depend not only on the person, but the activity.

\section{Do we benefit more from some activities than others?}

While we have largely lumped art, music, and literature together, treating these disciplines as interchangeable overlooks important distinctions (Shim et al., 2019). For instance, in a comparison of cultural activities, novels, cinema, and theater were among the best predictors of well-being; poetry and classical music less so (Grossi et al., 2010). We suggest that such differences are best understood in the context of the person by environment interactions discussed above. People's individual interests may lead them to seek out, enjoy, and benefit from different activities. As such, features of the activity may impact people's ability and motivation to engage successfully, including their accessibility (e.g., difficulty, cost, duration to subjective well-being payoffs), as well as social and cognitive fea- tures (e.g., primarily social vs solitary, requiring mentalizing or not) that impact the indirect benefits such activities are capable of conferring.

For instance, expressive writing has been found to have profound benefits for psychological well-being, presumably because autobiographical narratives necessarily entail deep processing and reappraisal of life events that lend itself to meaning-making (King, 2001; Pennebaker, 1997). Likewise, Noice and colleagues (2013) find that drama acting exercises confer well-being benefits above and beyond that of arts appreciation or singing interventions, in part because they require navigating complex simulated social interactions. And in reading, genre matters; many documented social cognitive benefits are restricted to literary fiction, presumably because fiction provides practice using socialcognitive processes to comprehend social information in a way that non-fiction typically does not.

Whether discipline matters depends, ultimately, on why the humanities are beneficial for subjective wellbeing. If the humanities increase well-being primarily by providing rich, interesting experiences, what matters most may be not the type of experience in question but people's subjective enjoyment of it. If so, any of the humanities may do the trick, as long as people are motivated and able to enjoy them. In contrast, if increases in well-being are driven indirectly via their social and cognitive benefits, then disciplines that maximize those benefits may be most beneficial. More evidence is needed to disentangle these theoretical mechanisms, and determine which better accounts for the relationship between the humanities and psychological wellbeing.

\section{Summary}

There is growing evidence the humanities make people's lives psychologically richer and happier, both via their direct and indirect effects on emotion and cognition. As in the classic proverb, "Give a man a fish and you feed him for a day; teach a man to fish and you feed him for a lifetime," the humanities offer a powerful potential tool for building better lives, providing people the lasting cognitive and social skills that form the building blocks of a rich, happy, and meaningful life. 


\section{References}

Blessi, G. T., Grossi, E., Sacco, P. L., Pieretti, G., \& Ferilli, G. (2014). Cultural participation, relational goods and individual subjective well-being: some empirical evidence. Review of Economics \& Finance, 4, 33-46.

Boothby, E. J., Clark, M. S., \& Bargh, J. A. (2014). Shared experiences are amplified. Psychological Science, 25, 2209-2216.

Brajša-Žganec, A., Merkaš, M., \& Šverko, I. (2011). Quality of life and leisure activities: How do leisure activities contribute to subjective well-being? Social Indicators Research, 102, 81-91.

Bryson, A., \& MacKerron, G. (2016). Are you happy while you work?. The Economic Journal, 127, 106-125.

Clift, S., Skingley, A., Coulton, S., \& Rodriguez, J. (2012). A controlled evaluation of the health benefits of a participative community singing programme for older people (Silver Song Clubs). Sidney De Haan Research Centre for Arts and Health, Canterbury Christ Church University, Folkestone, Kent, UK.

Coubard, O. A., Duretz, S., Lefebvre, V., Lapalus, P., \& Ferrufino, L. (2011). Practice of contemporary dance improves cognitive flexibility in aging. Frontiers in Aging Neuroscience, 3, 13.

Csikszentmihalyi, M. (2000). Beyond boredom and anxiety: Experiencing flow in work and play.

San Francisco: Jossey-Bass.

Csikszentmihalyi, M., \& Hunter, J. (2003). Happiness in everyday life: The uses of experience sampling. Journal of Happiness Studies, 4, 185-199.

Csikszentmihalyi, M., Rathunde, K., \& Whalen, S. (1997). Talented teenagers: The roots of success and failure. Cambridge University Press.

Dodell-Feder, D., \& Tamir, D. (2018). Fiction Reading Has a Small Positive Impact on Social Cognition. Journal of Experimental Psychology: General, 147, 1713-1727.

Ferguson, C. J. (2014). Is reading "banned" books associated with behavior problems in young readers? The influence of controversial young adult books on the psychological wellbeing of adolescents. Psychology of Aesthetics, Creativity, and the Arts, 8 , 354-362.

Grossi, E., Sacco, P. L., Blessi, G. T., \& Cerutti, R. (2011). The impact of culture on the individual subjective well-being of the Italian population: an exploratory study. Applied Research in Quality of Life, 6, 387-410.

Hand, C. (2018). Do the arts make you happy? A quantile regression approach. Journal of Cultural Economics, 42, 271-286.

Hartung F., Withers P., Hagoort P. and Willems R.M. (2017). When Fiction Is Just as Real as Fact: No Differences in Reading Behavior between Stories Believed to be Based on True or Fictional Events. Front. Psychol. 8:1618.

Hoven, E. Van Den, Hartung, F., Burke, M., \& Willems, R. M. (2016). Individual Differences in Sensitivity to Style During Literary Reading : Insights from Eye-tracking, Collabra, 2, 1-16.

Karpavičiūtè, S., \& Macijauskienè, J. (2016). The impact of arts activity on nursing staff well-being: An intervention in the workplace. International Journal of Environmental Research and Public Health, 13, 435.

Kattenstroth, J. C., Kalisch, T., Holt, S., Tegenthoff, M., \& Dinse, H. R. (2013). Six months of dance intervention enhances postural, sensorimotor, and cognitive performance in elderly without affecting cardio-respiratory functions. Frontiers in Aging Neuroscience, $5,5$.
Kim, S., \& Kim, H. (2009). Does cultural capital matter?: Cultural divide and quality of life. Social Indicators Research, 93(2), 295-313.

Kim, S. K. (2013). A randomized, controlled study of the effects of art therapy on older Korean-Americans' healthy aging. Arts in Psychotherapy, 40, 158-164.

King, L. A. (2001). The health benefits of writing about life goals. Personality and Social Psychology Bulletin, 27, 798-807.

Mar, R. A., \& Oatley, K. (2008). The Function of Fiction is the Abstraction and Simulation of Social Experience. Perspectives on Psychological Science, 3, 173-192.

Marsh, K., \& Bertranou, E. (2012). Can subjective well-being measures be used to value policy outcomes? The example of engagement in culture. Cultural Trends, 21, 299-310.

Michalos, A. C., \& Kahlke, P. M. (2008). Impact of arts-related activities on the perceived quality of life. Social Indicators Research, 89, 193-258.

Michalos, A. C., \& Kahlke, P. M. (2010). Arts and the perceived quality of life in British Columbia. Social Indicators Research, 96, 1-39.

Morinville, A., Miranda, D., \& Gaudreau, P. (2013). Music listening motivation is associated with global happiness in Canadian late adolescents. Psychology of Aesthetics, Creativity, and the Arts, 7, 384.

Morrow, D. G., Leirer, V. O., and Altieri, P. A. (1992). Aging, expertise, and narrativeprocessing. Psychology and Aging, 7, 376388.

Noice, T., Noice, H., \& Kramer, A. F. (2013). Participatory arts for older adults: A review of benefits and challenges. The Gerontologist, 54, 741-753.

Noice, H., Noice, T., \& Staines, G. (2004). A short-term intervention to enhance cognitive and affective functioning in older adults. Journal of Aging and Health, 16, 1-24.

Oatley, K. (1999). Why fiction may be twice as true as fact: Fiction as cognitive and emotional simulation. Review of general psychology, 3, 101-117.

Oatley, K. (2016). Fiction: Simulation of Social Worlds. Trends in Cognitive Sciences, 20, 618-628.

Oishi, S., \& Axt, J. (2019). A psychologically rich listening experience. A paper in preparation.

Oishi, S., \& Choi, H. (2019). What is a psychologically rich experience? An exploration of visual images. A paper in preparation.

Oishi, S., Choi, H., Buttrick, N., Heintzelman, S. J., Kushlev, K., Westgate, E. C., ... \& Ng, B. W. (2019). The Psychologically Rich Life Questionnaire. Journal of Research in Personality.

Oishi, S., Choi, H., Liu, A., \& Kurtz, J. (2019). A psychologically rich life: A consistent outcome of study abroad. A paper under review.

Pennebaker, J. W. (1997). Writing about emotional experiences as a therapeutic process. Psychological Science, 8, 162-166.

Puetz, T. W., Morley, C. A., \& Herring, M. P. (2013). Effects of creative arts therapies on psychological symptoms and quality of life in patients with cancer. JAMA Internal Medicine, 173, 960969.

Recht, D. R. and Leslie, L. (1988). Effect of prior knowledge on good and poor readers' memory of text. Journal of Educational Psychology, 80, 16-20.

Siedlecka, E., \& Denson, T. F. (2019). Experimental methods for inducing basic emotions: A qualitative review. Emotion Review, 11, 87-97.

Silvia, P. J. (2005). What is interesting? Exploring the appraisal structure of interest. Emotion, 5, 89-102.

Silvia, P. J. (2006). Exploring the psychology of interest. New York: Oxford University Press. 
Steger, M. F., Shim, Y., Rush, B. R., Brueske, L. A., Shin, J. Y., \& Merriman, L. A. (2013). The mind's eye: A photographic method for understanding meaning in people's lives. The Journal of Positive Psychology, 8, 530-542.

Tay, L., Pawelski, J. O., \& Keith, M. G. (2018). The role of the arts and humanities in human flourishing: A conceptual model. The Journal of Positive Psychology, 13, 215-225.

van Peer, W., Mentjes, A., \& Auracher, J. (2007). Does reading literature make people happy? In C. Martindale, P. Locher, \& V. M. Petrov (Eds.), Evolutionary and neurocognitive approaches to aesthetics, creativity, and the arts (pp. 47-64). Amityville, NY: Baywood Publishing.

Vessel, E. A., Starr, G. G., \& Rubin, N. (2012). The brain on art: Intense aesthetic experience activates the default mode network. Frontiers in Human Neuroscience, 6, 235-251.

Viding, C. G., Osika, W., Theorell, T., Kowalski, J., Hallqvist, J., \& Horwitz, E. B. (2015). "The Culture palette" - A randomized intervention study for women with burnout symptoms in Sweden. British Journal of Medical Practitioners, 8, 5-12.

Wang, M., \& Wong, M. S. (2014). Happiness and leisure across countries: Evidence from international survey data. Journal of Happiness Studies, 15, 85-118.
Westgate, E.C., \& Wilson, T.D. (2018). Boring thoughts and bored minds: The MAC model of boredom and cognitive engagement. Psychological Review, 125, 689-713.

Wheatley, D., \& Bickerton, C. (2017). Subjective well-being and engagement in arts, culture and sport. Journal of Cultural Economics, 41, 23-45.

Wheatley, D., \& Bickerton, C. (2019). Measuring changes in subjective well-being from engagement in the arts, culture and sport. Journal of Cultural Economics, 1-22.

Wilkins, R. W., Hodges, D. A., Laurienti, P. J., Steen, M., \& Burdette, J. H. (2014). Network science and the effects of music preference on functional brain connectivity: From Beethoven to Eminem. Scientific Reports, 4, 1-7.

Willems, R. M., \& Hartung, F. (2019). Engaging regularly with fiction influences connectivity in cortical areas for language and mentalizing. Unpublished manuscript.

Zhang, X., Yu, H. W., \& Barrett, L. F. (2014). How does this make you feel? A comparison of four affect induction procedures. Frontiers in Psychology, 5, 689. 